Revue d'histoire de l'Amérique française

REVUE D.HISTOIRE DE L'AMÉRIQUE FRANÇAISE

\title{
Le Québec et le plébiscite de 1942 sur la conscription
}

\section{J. L. Granatstein}

Volume 27, numéro 1, juin 1973

URI : https://id.erudit.org/iderudit/303231ar

DOI : https://doi.org/10.7202/303231ar

Aller au sommaire du numéro

Éditeur(s)

Institut d'histoire de l'Amérique française

ISSN

0035-2357 (imprimé)

1492-1383 (numérique)

Découvrir la revue

Citer cet article

Granatstein, J. (1973). Le Québec et le plébiscite de 1942 sur la conscription.

Revue d'histoire de l'Amérique française, 27(1), 43-62.

https://doi.org/10.7202/303231ar d'utilisation que vous pouvez consulter en ligne.

https://apropos.erudit.org/fr/usagers/politique-dutilisation/ 


\title{
LE QUEBBEC ET LE PLÉBISCITE DE 1942 SUR LA CONSCRIPTION *
}

\author{
J. L. Granatstein \\ Département d'histoire \\ York University
}

Vers la fin de 1941, on pouvait sentir au Canada un climat de grande frustration. Après deux années de guerre on avait l'impression qu'aucun résultat tangible ne venait justifier, aux yeux du public, tous les efforts consentis jusqu'à ce jour. En plus de l'augmentation des impôts, on venait d'établir un système de contrôle des prix et des salaires, et les gens étaient terriblement las. Pis encore, il y avait bien peu de victoires à porter au crédit des alliés. Les Russes lâchaient pied en désordre devant les panzers nazis, la campagne d'Afrique était encore une série de désastres et les Japonais envisageaient de porter la guerre dans le Pacifique. Les troupes canadiennes n'avaient pas encore participé à des combats d'importance; l'effort le plus important était le plan d'entraînement aérien du Commonwealth britannique qui, bien qu'utile, n'avait rien de spectaculaire; seule la modeste marine royale du Canada prenait tous les jours une part active au combat. C'était une drôle de guerre.

C'est dans ces circonstances déprimantes que, pour la première fois depuis le début de la guerre, on posa sérieusement le problème de la conscription pour servir dans les forces armées outre-mer. La conscription pour service militaire au Canada, qui entra en vigueur vers la fin de 1940, avait été autorisée, presque sans opposition, par la loi de la mobilisation des ressources nationales du mois de juin précédent. Mais à la fin de 1941 se dessinait un mouvement d'opinion de plus en plus important au Canada anglais exigeant un "total war effort", soit la conscription totale de la main-d'œuvre pour les forces armées. A la tête de ce mouvement d'opinion, on retrouve Arthur Meighen, l'auteur de la loi de la conscription de 1917 et deux fois premier ministre conservateur pendant les années vingt. Pour Meighen et ses amis, comme pour des milliers de Canadiens, aucune guerre ne pouvait être gagnée sans cet effort total. Lorsqu'il fut choisi comme chef du parti conservateur en novem-

* Je suis très reconnaissant aux exécuteurs testamentaires de la succession Mackenzie King pour leur gracieuse permission de consulter ses archives privées dont son journal personnel, en vue de la préparation d'une biographie de King, 1939-1945. 
bre 1941, la conscription devint une fois de plus le problème majeur au Canada.

Pour les libéraux et surtout pour le premier ministre Mackenzie King, il ne pouvait y avoir de question plus explosive. Comment oublier "300 years of history and 25 years of politics" 1, 1917, Laurier, la division du parti et du pays. King croyait sincèrement que seul le parti libéral pouvait sauvegarder l'unité canadienne pendant toute la guerre. Il était conscient qu'il lui faudrait manœuvrer très habilement pour assurer la survie de son gouvernement, pour éviter de placer dans une position intenable les ministres du cabinet et les députés libéraux du Québec et pour empêcher le Canada anglais d'imposer à la nation le service militaire obligatoire. Le problème se compliqua avec la disparition d'Ernest Lapointe, son lieutenant qui jouissait de la confiance et du respect dans toutes les régions du Canada.

Nous verrons comment King, entre novembre 1941 et avril 1942, put réussir à atteindre quelques-uns de ses objectifs, grâce à une tactique avisée et habile. Cependant, il ne parvint pas à obtenir l'appui du Québec lors du plébiscite sur la conscription en avril 1942. Une des causes principales de cette défaite, comme cette étude le démontrera, fut la campagne populaire organisée par un groupe de pression remarquable, la Ligue pour la défense du Canada.

King, pour éviter la conscription, dut affronter l'opposition à l'intérieur et à l'extérieur de son cabinet. La plus grande menace extérieure provenait d'Arthur Meighen, appuyé par son parti et par la presse conservatrice ${ }^{2}$; tandis qu'au sein du cabinet, les principaux défenseurs de la conscription étaient le colonel J. L. Ralston, avocat montréalais d'origine néo-écossaise, et Angus L. MacDonald, premier ministre de la Nouvelle-Ecosse, qui, en 1940, avait répondu à l'appel de King d'entrer au cabinet. MacDonald, en qualité de ministre de la Marine, collaborait étroitement avec son ami Ralston, le ministre de la Défense nationale. Tous deux avaient pleinement conscience des besoins des forces armées et, à la fin de 1941, convaincus que le Canada devait faire un effort suprême pour augmenter ses effectifs militaires, ils étaient prêts à supporter la conscription

1 Archives publiques du Canada [APC], Fonds Brooke Claxton, t. 21, Claxton à E. Armstrong, 1er mai 1942.

2 Les conservateurs et la conscription ont été étudiés à fond dans J. L. Granatstein, The Politics of Survival: The Conservative Party of Canada -1939-1945 (Toronto, 1967). 
si nécessaire. King se devait de pousser l'effort de guerre de façon à convaincre ses ministres, Ralston et MacDonald, voire même le Canada anglais, que le pays s'acquittait pleinement de ses responsabilités. En même temps, il devait respecter sa promesse maintes fois réitérée qu'il n'y aurait pas de conscription.

Les ministres de la Défense nationale voulaient porter à cinq divisions le corps d'armée canadien stationné en Angleterre, ce qui, en 1942, nécessitait un énorme recrutement. Une étude de la main-d'œuvre commandée par le cabinet démontrait clairement qu'on ne pouvait compter sur les services de plus de 600,000 hommes dans tout le pays et que, de ce nombre, 200,000 étaient requis par l'Aviation et la Marine. On craignait qu'à cause des exigences techniques, surtout dans l'Aviation, peu de Canadiens français pourraient y être admis. L'éducation classique n'était pas sans valeur, mais elle ne développait guère les aptitudes techniques nécessaires pour l'entretien des avions. ${ }^{3}$

Ce problème de main-d'œuvre retint l'attention des membres du cabinet pendant plusieurs jours. Les attaques japonaises dans le Pacifique, le 7 décembre, rendirent plus impérieux le besoin d'hommes pour assurer la défense du territoire canadien et l'expédition de fournitures essentielles des Etats-Unis vers l'Angleterre. Ralston en profita pour affirmer à nouveau qu'il favoriserait la conscription si elle était nécessaire pour recruter un nombre d'hommes suffisant ${ }^{4}$; mais, d'autre part, il semblait hésité à conscrire un trop grand nombre de Canadiens français. Ralston avait discuté de cette question, le 7 décembre, avec l'éditeur Victor Sifton qui occupait le poste de Master General of the Ordnance au quartier général de la Défense nationale. Sifton raconta la conversation à Grant Dexter du Winnipeg Free Press :

When Victor pointed out that after deducting 200,000 English speaking for the air force and navy, this would leave 400,000 for the army but they would have to take one French Canadian for every English-speaking Canadian, Ralston protested. The army would not have masses of Quebeckers under any conditions: they would not be able to do anything with them

3 C. P. Stacey, Arms, Men and Governments: The War Policies of Canada, 1939-1945 (Ottawa, 1970), 403. - Queen's University, Fonds Grant Dexter, Mémorandum, 20 novembre 1942.

${ }^{4}$ Fonds Dexter, Mémorandum, 5 décembre 1941. Dexter avait des relations étroites avec plusieurs ministres du cabinet, plus spécialement avec T. A. Crerar. Ses mémoranda, envoyés régulièrement à son rédacteur en chef, J. W. Dafoe, du Winnipeg Free Press, sont une excellente source d'informations sûres concernant les personnalités et les événements mentionnés dans cet article. 
[dit Ralston]. There is only limited room in our army for these men. They can't speak English. We have no French $\mathbf{C}$ [sic] officers to handle them. Their fighting ability is questionable, etc., etc. Ralston said that conscription was necessary to get more men from the English-speaking provinces...5

On ne saurait dire si ces idées exprimées par Ralston correspondaient à une opinion mûrement réfléchie.

La pression augmentait aussi à l'extérieur du cabinet. Meighen réclamait avec force la conscription et la création d'un gouvernement de coalition ["national government"]. C'était à nouveau la politique de 1917. La presse consacrait de plus en plus d'espace aux idées de Meighen; les nouvelles désastreuses de la guerre n'aidaient en rien le gouvernement. Devant la pression croissante des forces politiques, King jugea qu'il se devait de réagir. Dès le 18 novembre, T. A. Crerar, ministre des Mines et des Ressources, conjecturait que King tiendrait un plébiscite sur la conscription et il avouait à l'ubiquiste Dexter du Winnipeg Free Press, que le premier ministre "will take the position that if conscription carries he will retire and some other leader will have to come forward to carry it out" ${ }^{6}$. Pendant un mois King laissa cette idée en suspens, mais le 18 décembre, il déclara au cabinet que "this government must be kept in power for the good of the country. Conscription is likely to divide the Government. Therefore let us take a plebiscite and be guided by it." 7 En plus, comme il le nota dans son journal, tout compte fait, un plébiscite serait utile du point de vue politique, car ainsi les Tories ne pourront profiter de la nouvelle session du parlement, en janvier 1942, pour réclamer un appel au peuple sur la question de la conscription, et auquel King aurait pu difficilement s'opposer. ${ }^{8}$ King pensa d'abord à formuler la question du plébiscite de la manière suivante: "Are you in favour of the government's having power to send men beyond Canada ?". Une suggestion plus simple était notée dans le journal d'Angus MacDonald: "Are you in favour of conscription?" 9. Bientôt d'autres ministres imaginèrent de nouvelles suggestions, en particulier Ian Mackenzie, ministre des Pensions, qui en envoya huit au ministre de l'Aviation, Chubby Power, responsable des aspects pratiques

5 Ibid., 9 décembre 1941.

6 Ibid., 18 novembre 1941.

7 Archives publiques de la Nouvelle-Ecosse, Fonds A. L. MacDonald, Journal, 18 décembre 1941.

8 J. W. Pickersgill, The Mackenzie King Record, I: 1939-1944 (Toronto, 1960) : 313 .

9 Fonds A. L. MacDonald, Journal, 18 décembre 1941. 
de la tenue d'un plébiscite. ${ }^{10}$ Bien que rien ne fût décidé, il apparaissait évident qu'un plébiscite offrait des avantages. En plus de donner un sursis, cela couperait l'herbe sous le pied de Meighen et des conscriptionnistes et, finalement, sauvegarderait l'unité des ministres canadiens-français. Selon Crerar, le projet de plébiscite avait l'appui de ceux-ci, sauf celui de Cardin, ministre des Travaux publics, qui avait pris la relève de Lapointe comme chef de file de l'aile québécoise. Le premier ministre, ajoute Crerar, était en consultation avec d'autres personnages importants, y compris le premier ministre du Québec, Adélard Godbout, et le cardinal Villeneuve. ${ }^{11}$

Le long débat au sujet des besoins de la main-d'œuvre pour l'armée se prolongea jusqu'au 5 janvier 1942. King rallia les membres de son cabinet aux propositions ambitieuses de Ralston en ajoutant "that we believed conscription was unnecessary; that the programme represented an all-out effort; that we believed this programme could be carried out without resort to conscription" 12 . Ce compromis politique pouvait préserver temporairement l'unité du cabinet, mais sans pour autant convaincre Ralston et MacDonald que la conscription n'était pas le meilleur moyen de recruter des hommes. Il en fallait plus pour apaiser Meighen. Un plébiscite était quand même un stratagème utile.

En fait, selon T.A. Crerar, qui fut encore une fois le premier à le noter, les ministres québécois partagèrent de plus en plus l'opinion de King. L'Acadien J.-E. Michaud, ministre des Pêcheries, crut qu'un plébiscite offrirait une solution pour le Québec:

He told me that a few weeks ago he had had a talk with the Cardinal [Villeneuve] and that the Cardinal feels strongly about the war and the need for an Allied victory; that he expressed the opinion on the conscription issue that, if the Government got released from its pledge by a referendum and, after this release, if King were to announce that the Government was prepared to put in conscription should it prove necessary to maintain our forces overseas, he would support it.

Mais, ajouta Michaud, ce serait insuffisant pour s'assurer l'appui de Québec de simplement laisser au gouvernement le soin de décider de sa propre initiative que, si le système volontaire ne réussissait pas, la conscription serait imposée. "It would be necessary to show Quebec clearly what the view was of the rest

${ }^{10} \mathrm{Ibid}$. et Queen's University, Fonds C. G. Power, Mackenzie à Power, 19 décembre 1941.

11 Fonds Dexter, Mémorandum, 22 décembre 1941.

12 Pickersgill, ibid., I: 335. 
of Canada on the matter; that they attach a great deal of importance to the pledges which have been given." 13 P.-J.-A. Cardin, devenu le personnage clé, attachait lui aussi beaucoup d'importance à ces promesses. Crerar lui rendit visite le 16 janvier et, au cours d'un long entretien, il se servit des arguments utilisés par Michaud. A la suite de cette rencontre, il nota: "I got the impression from him that personally he would not object to this course. Before leaving he told me that he was not without some considerable hope that things might so arrange themselves in Quebec that there would be a minimum of criticism of a referendum, and went on to say that in times like these it was entirely reasonable that a government should have freedom of action..."14 Finalement, Cardin se laissa convaincre, et après beaucoup de tergiversations, le premier ministre décida d'annoncer le plébiscite dans le Discours du Trône à l'ouverture de la session, le 22 janvier. Pendant trois réunions du cabinet, les 19,20 et 21 janvier, on discuta à fond la rédaction finale du passage relatif au plébiscite qui se lirait ainsi: "My ministers accordingly will seek, from the people, by means of a plebiscite, release from any obligation arising out of any past commitments restricting methods of raising men for military service." La date du plébiscite fut fixée au 27 avril 1942.

Les raisons qui ont motivé la décision de King de tenir un plébiscite sont complexes. Lors d'une série de réunions avec des éditeurs québécois, au mois de mai, King rencontra Georges Pelletier du Devoir, qu'on ne saurait considérer comme un ami ou un partisan du gouvernement ou de ses politiques; il lui expliqua ses raisons de la manière suivante:

Il [King] continue en disant qu'en janvier 1942 [écrit Pelletier], la situation était en train de devenir la même [qu'en 1940]. Il était attaqué d'un côté par les conservateurs dans le pays et dans la presse tory de Toronto et d'ailleurs, qui réclamaient tous ensemble la conscription, bien qu'en février et mars 1940, ils n'en voulussent point. D'un autre côté, à la Chambre, M. King se trouvait à avoir affaire à Hanson et à son petit groupe de Tories qui, dit-il, menaçaient de répéter les tactiques de 1940, en même temps que Hepburn et les Deux Cents de Toronto insistaient pour avoir un effort de guerre total. De plus, la candidature Meighen était affichée depuis plusieurs semaines; Meighen devait remplacer Hanson 1942.

13 Queen's University, Fonds T. A. Crerar, Crerar à Dafoe, 8 janvier

14 Ibid., Mémorandum dicté par le ministre... le 16 janvier 1942. King et Cardin discutèrent à fond le sujet de façon à peu près identique, le 15 janvier de la même année, Pickersgill, ibid., I : 337. 
comme chef du parti tory, et il paraissait impossible d'admettre qu'il fut bon pour le Canada de laisser entrer Meighen à la Chambre. "J'ai travaillé toute ma vie, dit M. King, pour faire l'accord entre les deux races. On m'a accusé de faire des concessions à Québec; mais, en fait, pour avoir l'accord entre les deux grandes races du pays, il faut pratiquer une politique de concessions. Or Meighen, qui est l'âme damnée d'un groupe de Toronto, fanatique et d'esprit très étroit et très égoïste, aurait extrêmement compliqué la situation, à une période dangereuse, s'il fût entré aux Communes. Je ne pouvais tout de même pas décider de faire des élections générales comme en 1940, pour écraser les adversaires de tous les camps. J'avais déjà fait entendre à Calgary et à Victoria qu'il ne pouvait pas être question de conscription sans un appel au peuple. Or, s'il n'y avait pas d'élection, le seul moyen d'en appeler au peuple c'était un plébiscite. Cela s'imposait, parce que si je n'avais pas annoncé de plébiscite, il $y$ aurait eu toutes les chances du monde que Meighen revînt aux Communes et nous aurions alors perdu le temps de la Chambre dans des querelles interminables et des débats extrêmement acerbes et inutiles pour autant; car Meighen est d'une force considérable dans l'invective... En décidant le plébiscite et en l'annonçant avant l'élection de Meighen, j'ai cru que c'était une excellente façon de lui barrer la route..."

L'autre problème majeur qui le préoccupait, avoua King à Pelletier, était la force des arguments en faveur de la conscription:

“... devant l'insistance de tout un groupe qui réclamait la conscription, j'ai cru et je crois encore que le plébiscite était le meilleur moyen de remettre les choses dans l'état où elles devaient être. En effet, s'il n'y avait pas eu le plébiscite, la campagne en faveur de la conscription de la part des conservateurs et des feuilles tory aurait été en s'accroissant, et elle fut devenue un danger pour le Canada. Je me suis dit que si nous allions devant le peuple avec un plébiscite, nous obtiendrions l'avis de la masse des électeurs, la libération de nos promesses de 1939 et qu'ensuite il serait assez facile de démontrer au peuple qu'il n'y avait pas besoin de conscription, pendant plusieurs mois en tout cas, peut-être pas du tout." 15

Le raisonnement persuasif de King fut, par la suite, présenté au caucus des libéraux. Il fallut convaincre les députés

15 Fondation Lionel-Groulx, Fonds Georges-Pelletier, "Entrevue Georges Pelletier-King (26 mai 1942)", 9-11. - Dans son journal King nota à propos de Pelletier: "I like him very much"; et il ajouta "I think the conversation did good and that we will see results that will be helpful", APC, Fonds King, Journal, 26 mai 1942, 449-450. 
québécois qu'on devait libérer le gouvernement de ses promesses et, pour ce faire, Cardin était indispensable. A une réunion du caucus, le 22 janvier, il s'acquitta de sa tâche en racontant ses hésitations pour accepter l'idée d'un plébiscite. Le Québec avait bien accompli son devoir dans la guerre, mais, dit-il, "You English-speaking people are influenced not only by your judgment but by your sentiments; the call comes to your hearts from the Motherland from which you came. The French-Canadians have not that call; our decision comes from our minds; it arises from our judgment alone." Il conclut en disant à ses compatriotes: "I am going to vote for the release, and I am going to urge my fellow French-Canadians to do the same thing." Ce fut "an eloquent and moving appeal," 16 écrivit T. A. Crerar à son ami J. W. Dafoe à Winnipeg.

On ne savait si d'autres Québécois voudraient ou pourraient accepter ce point de vue. L'ambassadeur américain à Ottawa, Pierrepont Moffat, avait des relations très étendues et, immédiatement après l'annonce du plébiscite, il rapporta les dires de plusieurs Canadiens français: "We think that M. Lapointe would have agreed to this plebiscite." 17 Peut-être avaient-ils raison, mais quelques semaines plus tard, Moffat eut une conversation avec sir Eugène Fiset, lieutenant-gouverneur du Québec. Selon ce dernier, le "québécois moyen" se demandait: "Is it not strange that within a very few weeks of the death of Mr. Lapointe, Mr. King should be asking a release from his solemn pledges ?... He is asking for a release from his promise at a time when voluntary enlistments are good and getting better. Ergo, he must be made to feel that the Province is disappointed in his volte-face." 18 Moffat considérait que le gouvernement tardait trop longtemps à tenir le plébiscite. "This will allow the sores of national disunity to fester and if the present bitterness on the part of Ontario continues, there may be as much disunity resulting from a plebiscite as there would have been by completely ignoring Quebec." Bien qu'il partageât le point de vue des conscriptionnistes, il n'en reconnaissait pas moins que "their tactics ... are so short-sighted, so uncooperative and so insulting

16 Fonds Crerar, 23 janvier 1942. - Pickersgill, ibid., I: 341. - Plus tard Cardin dira à un député québécois qu'il favorisait le plébiscite parce que "il vaut mieux avoir la conscription avec $M$. King qu'avec un autre Hanson ou Meighen", Fondation Lionel-Groulx, Fonds Bloc Populaire canadien, dossier Honorable Cardin, Mémorandum, s.d. - Pour des renseignements sur les dissensions au caucus, voir MacDonald, Journal, 28 janvier 1942.

17 Harvard University, Fonds Pierrepont Moffat, t. 47, Mémorandum d'une conversation avec M. Philippe Brais ..., 24 janvier 1942.

18 Ibid., Mémorandum, 19 février 1942. 
to anyone who happens to hold a different point of view that one's feelings of sympathy are obscured." 19

Certains adversaires de la conscription se montraient aussi sans tact et étroits d'esprit dans leurs observations. Dans un discours à Montréal, le 26 janvier, le premier ministre Godbout, sous le coup de la provocation et du harcèlement d'un auditoire nationaliste, déclara que "le service obligatoire pour outre-mer, dans le moment, serait un crime..." Il enchaîna en disant que Mackenzie King savait que l'Angleterre avait besoin d'armes et non d'hommes et qu'il n'y enverrait plus de soldats. ${ }^{20}$ Naturellement, ce discours gêna profondément le gouvernement fédéral et, aux reproches que lui fit King, Godbout ne put que répondre qu'il avait improvisé et qu'il ne se rappelait pas ses mots exacts. ${ }^{21}$

Parmi ceux qui avaient été les plus affectés par les implications des remarques de Godbout, se trouvaient Ralston et MacDonald. Les deux ministres de la Défense nationale rendirent visite à King au Laurier House le 31 janvier pour discuter de la question. A cette occasion, Ralston invoqua le délai causé par le plébiscite et demanda l'autorisation d'annoncer publiquement que si la conscription devenait nécessaire avant la tenue du plébiscite, elle serait mise en application. La réponse de King fut reproduite dans les notes de la conversation qu'il eut avec MacDonald :
P.M. said the question had to be viewed as a whole. It was a mistake to urge conscription just for conscription's sake. We must be sure we would get more men under conscription than without it. We must fill the needs of industry, farming, home defence, as well as the needs of the armed forces... Conditions in this country might get so bad that no one could govern the country. If you had to use machine guns, what would be the use of conscription? He had denied the Godbout statement. Surely his colleagues would believe him.

Ralston jugea que la réponse du premier ministre éludait le véritable problème. Ce qui inquiétait les deux ministres, c'était de savoir ce qu'il adviendrait si le peuple votait oui lors du plébiscite. King répliqua que la décision serait prise "considering everything", en expliquant qu'il faudrait tenir compte de l'opi-

19 Ibid., Mémorandum d'une conversation avec J. W. McConnell..., 24 janvier 1942. - Pour la réaction au Canada anglais à la proclamation du plébiscite, voir Granatstein, The Politics of Survival ..., 105-107.

20 Robert Rumilly, Histoire de la province de Québec - XXXIX: La guerre de 1939-1945 - Le plébiscite (Montréal, 1969): 184. - Montreal Star, 27 janvier 1942.

21 Fonds Dexter, Mémorandum, 27 janvier 1942. 
nion de l'ensemble du pays et non seulement de celle du Québec. Bien qu'insatisfait de cette explication, MacDonald n'insista pas. Il termina sa description de l'entretien en suspectant que la réaction de King aux résultats du plébiscite, quels qu'ils fussent, pourrait être que "Conscription might give you a few more men here and there, but it would create a terrible situation in the country and consequently will not be worth while." 22

La proclamation du plébiscite eut pour conséquence heureuse, aux yeux de toutes les factions dans le cabinet et au Québec, de provoquer la défaite retentissante d'Arthur Meighen dans une élection complémentaire à Toronto le 9 février. Cette défaite fut causée, au moins en partie, par la façon dont le plébiscite privait Meighen du thème majeur de sa campagne. ${ }^{23}$ Les victoires respectives de Humphrey Mitchell, le nouveau ministre du Travail, et de Louis Saint-Laurent, le nouveau ministre de la Justice, dans des élections complémentaires tenues à Welland (Ontario) et à Québec-Est, furent d'autant plus importantes que Mitchell avait dû faire face à la vive opposition du premier ministre Hepburn et les libéraux de l'Ontario, et que Saint-Laurent, de son côté, avait dû affronter celle des nationalistes. Ces résultats laissaient croire que l'opposition au gouvernement, tant au Québec qu'en Ontario, n'était pas aussi forte qu'on l'avait craint. Le lendemain des élections, T. A. Crerar écrivait: "The time between now and the taking of the plebiscite offers some time for passions ... to cool. I expect the debate will collapse in a few days..." ${ }^{24}$

Si Crerar avait raison de penser ainsi pour le Canada anglais, par contre son pronostic était complètement faux pour le Québec où, pouvait noter le jeune député montréalais Brooke Claxton, le sentiment contre la conscription était toujours aussi marqué:

99.9\% of the people in Quebec are against conscription and have been right along. Recently a Gallup Poll on this matter showed a vote of two to one against in Quebec. I thought then and know now that it was much higher. Despite active searching I do not think that I have found a French-Canadian who is in favour of conscription. You hear talk in places like

22 APC, Fonds J. L. Ralston, Mémorandum, "Conversation Ralston and I had with P.M...." - MacDonald, Journal, 31 janvier 1942.

23 J. L. Granatstein, "The York South By-Election of February 9, 1942: A Turning Point in Canadian Politics", Canadian Historical Review, XLVIII (juin 1967) : 142-158.

24 APC, Fonds J. W. Dafoe, Crerar à Dafoe, 10 février 1942. Cependant un sondage fait le 14 février révéla que $54 \%$ des gens croyaient que le gouvernement avait tort de tenir un plébiscite et seulement $31 \%$ approuvaient cette décision, Public Opinion Quarterly (été 1942) : 312. 
Westmount, Toronto, Hamilton and Winnipeg about French Canada being in favour of conscription but you cannot run anyone who is to earth and you certainly cannot get them out into the open. ${ }^{25}$

Cependant il n'y avait aucun problème à trouver des anticonscriptionnistes. Après les événements de 1917 et ceux des années suivantes pendant lesquelles le parti libéral avait fait maintes campagnes contre les "bloody hands" de Meighen, chaque Québécois était anticonscriptionniste convaincu et la Ligue pour la défense du Canada était son porte-parole.

Les origines de cette LPDC sont un peu obscures. Selon André Laurendeau, le premier secrétaire de la Ligue, plusieurs personnes eurent simultanément l'idée d'une organisation pour mobiliser l'opposition des anticonscriptionnistes lors du plébiscite. ${ }^{26}$. Robert Rumilly, de son côté, attribue cette initiative à un groupe de jeunes hommes dont Michel Chartrand et Roger Varin que l'abbé Groulx engagea à aller voir Laurendeau et Paul Gouin, l'ancien chef de l'Action libérale nationale. De toute façon on organisa la Ligue qui reçut son nom lors d'une réunion chez Gouin ${ }^{27}$ qui eut lieu vraisemblablement avant la fin de janvier 1942. L'objectif de la LPDC fut de réunir les représentants officiels des grandes organisations publiques et d'établir entre elles des liens étroits sans toutefois leur faire perdre leur liberté d'action. Une fois la Ligue établie, ses directeurs furent choisis parmi les dirigeants de l'Union catholique des cultivateurs, du Conseil des syndicats catholiques de Montréal, de la Société Saint-Jean-Baptiste de Montréal, des Voyageurs de commerce et de divers mouvements de jeunesse. Laurendeau lui-même représentait la Ligue d'Action nationale. Le Dr J.-B. Prince, "un vétéran du bourassisme" ${ }^{28}$, fut choisi président de la LPDC, mais ses véritables leaders étaient Maxime Raymond, député libéral de Beauharnois-Laprairie, et Georges Pelletier, directeur du Devoir et successeur de Bourassa à la tête du principal organe nationaliste au Québec. ${ }^{29} \mathrm{~A}$ ces deux noms, on doit ajouter celui de Laurendeau qui, à titre de secrétaire, était responsable des opérations quotidiennes de la LPDC et, de ce fait, se trouvait à définir certaines des politiques du mouvement.

25 Fonds Claxton, t. 53, Claxton à J. M. Macdonnell, 18 février 1942. 1962), 81 .

26 André Laurendeau, La crise de la conscription, 1942 (Montréal,

27 Rumilly, ibid. XXXIX : 187.

28 Laurendeau, ibid., 82.

29 Ibid. 
La LPDC publia son manifeste dans le numéro de janvier de L'Action nationale et le diffusa largement à travers la province sous forme de tract imprimé en anglais et en français. L'argumentation était sans détour: il faut voter non au plébiscite. Pourquoi: "Parce que nul ne demande d'être relevé d'un engagement s'il n'a déjà la tentation de le violer, et parce que, de toutes les promesses qu'il a faites au peuple du Canada, il n'en reste qu'une que King voudrait n'être plus obligé de tenir: la promesse de ne pas conscrire les hommes pour outre-mer." 30

Le plébiscite fit l'objet d'une critique plus approfondie de la part de Maxime Raymond. Dans un discours à la Chambre des Communes, le 5 février, Raymond commença par souligner l'ampleur du programme de guerre que le gouvernement exposait dans le Discours du Trône. Ce programme proposait un prêt de sept cents millions de dollars et un don d'un milliard de dollars à l'Angleterre, un budget pour la guerre de trois milliards de dollars et le plébiscite. C'était "un effort de guerre national poussé jusqu'à l'extrême limite", trop considérable pour un petit pays. La proposition de la tenue d'un plébiscite était encore plus scandaleuse car elle violait le pacte qu'on avait fait le 9 septembre 1939 et renouvelé à l'élection de 1940. N'est-ce pas Ernest Lapointe qui disait à la Chambre et au pays ce 9 septembre qu'il était "autorisé par [ses] collègues de la province de Québec dans le cabinet... à déclarer que nous ne consentirons jamais à la conscription, que nous ne serons jamais membres d'un gouvernement qui essayera d'appliquer la conscription et que nous n'appuierons jamais un tel gouvernement. Est-ce assez clair ?" La suite du discours de Lapointe, que rapporta Maxime Raymond, était aussi ferme dans son énoncé: "Pourvu que l'on comprenne bien ces points, avait dit Lapointe, nous sommes prêts à offrir nos services sans restriction et à vouer le meilleur de nous-mêmes au succès de la cause que nous avons tous à cœur." Pour Raymond, une seule interprétation possible: "Participation sans conscription". Le pacte était formel et le plébiscite en constituait une violation. On avait promis au Québec qu'il n'y aurait pas de conscription et voilà que maintenant on demandait au pays tout entier de libérer le gouvernement de cette promesse faite au Canada français. ${ }^{31}$ Le discours de Raymond servit de base au plaidoyer dressé par la LPDC. Canada.

30 Fondation Lionel-Groulx, Fonds de la Ligue pour la défense du

31 Fondation Lionel-Groulx, Fonds Maxime-Raymond, adresse du 5 février 1942. - King n'acceptait pas que la promesse avait été faite seulement au Québec: "... that was nonsense as most of my speeches had been on the radio to the entire country...", King, Journal, 14 mai 1942. 
Nantie de bons arguments, la Ligue orienta ses efforts du côté de l'organisation et de la propagande. Les tactiques utilisées furent celles d'un groupe de pression conventionnel. On organisa des assemblées publiques, on fit des quêtes, on vendit des cartes de membre à un dollar chacune et on sollicita de plus généreuses contributions de la part des gens riches et des organisations. Un véritable déluge de tracts furent distribués partout dans la province, de même qu'au Nouveau-Brunswick et dans le nord de l'Ontario. On remit des communiqués de presse aux journaux et aux postes de radio. La machine fonctionnait rondement, ce qui était exceptionnel pour ce genre d'organisation ad hoc.

Même si la Ligue disposait de peu d'argent elle fit preuve d'efficacité. A en juger par les états de comptes encore existants de la LPDC les contributions individuelles étaient modestes, soit en moyenne deux dollars; ${ }^{32}$ quelques-unes des réunions ne rapportèrent que peu ou pas de revenus. ${ }^{33} \mathrm{~A}$ certains moments, Laurendeau se vit dans l'obligation d'implorer les directeurs de journaux de faire preuve d'équité en renseignant les lecteurs sur les activités de la Ligue qui se trouvait dans l'impossibilité de concurrencer le gouvernement disposant d'un énorme budget de publicité, car, écrivait-il au journal Le Droit, à Ottawa: "La Ligue est trop pauvre..." 34

De plus, la presse en général se montrait très prudente quand il s'agissait de traiter des activités de la Ligue. Si le silence des journaux anglophones, tels la Montreal Gazette, le Montreal Star et le Quebec Chronicle Telegraph, était prévisible, les réticences de la presse française à prendre une quelconque position durent contrarier la Ligue. Cela surprit certainement Mackenzie King et le gouvernement libéral qui s'inquiétaient de ne pas trouver dans La Presse, Le Canada, Le Soleil et L'Evénement l'appui attendu en faveur de leur position à propos du plébiscite. La propagande du gouvernement fut sérieusement compromise, semble-t-il, par la rivalité entre les partisans de Cardin et ceux du premier ministre Godbout. ${ }^{35}$ Le Jour, dirigé par Jean-Charles Harvey, fut pratiquement le seul journal à accuser les membres de la Ligue d'être des "trâ̂tres". ${ }^{36} L e$ Devoir, de son côté, fut l'unique journal important à défendre

32 Fondation Lionel-Groulx, Fonds LPDC, Livre de caisse 1942-1943. 33 Ibid., J.-P. Cloutier à Laurendeau, 6 mai 1942, précisant qu'une réunion le 24 avril n'avait rapporté que $\$ 6.45$.

34 Ibid., Laurendeau à Camille L'Heureux, 17 avril 1942. 422 .

35 King, Journal, 19 mars 1942, 245; 12, 13, 14 mai 1942, 412-413, 415,

36 Marcel-Aimé Gagnon, Jean-Charles Harvey, précurseur de la révolution tranquille (Montréal, 1970), 194. 
avec enthousiasme le non. ${ }^{37}$ Quant à la radio, la LPDC ne pouvait s'y faire entendre, sauf pour les quelques périodes de temps louées à des postes privés. Radio-Canada, sous la pression du gouvernement, décida arbitrairement, durant la campagne du plébiscite, de réserver les ondes aux seuls partis politiques officiels. Cette tactique signifiait que seuls les partis représentés au parlement, qui tous demandaient de voter oui, seraient entendus. ${ }^{38}$

Malgré tout, les partisans du oui furent impuissants à empêcher la Ligue de transmettre son message. Généralement un public nombreux et enthousiaste assistait à ses assemblées. $\mathrm{Au}$ moins une fois, la LPDC attira environ dix mille personnes à un grand rassemblement tenu à Montréal. Les sections de la LPDC se multipliaient spontanément à travers la province, souvent avec l'assistance de la SSJB. ${ }^{39}$ Cette décentralisation n'affecta pas l'unité du mouvement. Pendant que le mouvement mettait sur pied une organisation pour couvrir le territoire de chaque bureau de scrutin, dans la ville de Montréal, ${ }^{40}$ des nationalistes prestigieux se joignaient au mouvement. A Québec, par exemple, René Chaloult et Philippe Hamel adhérèrent à la Ligue.

Chaloult ne tarda pas à se manifester en présentant à l'Assemblée législative une proposition en faveur du non. Faisant valoir que les législatures de l'Alberta et du Manitoba s'étaient prononcées pour le oui, Chaloult soutint que le Québec pouvait tout aussi légitimement exprimer son opinion. De toute évidence cette proposition embarrassait le gouvernement du premier ministre Godbout dont on n'avait pas oublié le discours du 26 janvier. Pendant quelque temps, il sembla que le cabinet allait tomber sur cette question, mais après de très fortes pressions sur les ministres et les députés libéraux, la proposition de Chaloult fut défaite. ${ }^{41}$ A la Chambre des Communes, JeanFrançois Pouliot proposa l'ajournement à six mois du projet de loi sur le plébiscite, ce qui fut facilement rejeté avec seulement treize votes favorables. Curieusement, deux de ces votes furent ceux de Tories de Toronto qui désiraient la conscription immédiate sans recours au plébiscite.42

37 Rumilly, ibid., XXXIX: 218. - Laurendeau, ibid., 97.

38 F. W. Peers, The Politics of Canadian Broadcasting (Toronto, 1969), 328-331. - Fondation Lionel-Groulx, Fonds LPDC, Laurendeau à Frigon, 4 avril 1942.

39 L.-A. Fréchette, "A chacun ses responsabilités", L'Action nationale, XIX (avril 1942) : 220-221.

20 avril 1942 .

40 Fondation Lionel-Groulx, Fonds LPDC, E. Simard à Laurendeau,

41 Rumilly, ibid., XXXIX : 212ss. — Laurendeau, ibid., 96-98.

42 Rumilly, ibid., XXXIX: 214. 
Tous ces efforts servirent à entretenir l'agitation dans l'opinion publique. La LPDC intensifia sa propagande à mesure qu'approchait le jour du scrutin. On ne cessait d'évoquer le souvenir d'Ernest Lapointe. Ainsi l'article de Georges Pelletier, qui parut dans Le Devoir du 21 février, sous le titre "Jamais, jamais ..... a dit Lapointe", fit l'objet d'une large diffusion sous forme de tract. ${ }^{43}$ Pour sa part, L'Aiglon, "l'organe libéral Dorchester-Bellechasse", publié à Saint-Joseph-de-Beauce, titra en gros caractères: "L'honorable Ernest Lapointe a dit: La conscription, jamais. Votons NON." ${ }^{44}$ Un autre thème populaire invitait à garder les hommes pour la défense du territoire. "Souvenons-nous de Hong-Kong"; "Souvenons-nous de l'Australie. Gardons nos conscrits chez nous." 45 On rappelait également les anciennes promesses de Mackenzie King que L'Action nationale citait in extenso. ${ }^{46}$ Cette même revue consacra des numéros presque entiers à attaquer la politique d'Ottawa et à expliquer vigoureusement et avec force détails "Pourquoi nous n'accepterons JAMAIS la conscription pour service outre-mer". ${ }^{47}$ Le plébiscite semblait créer un sentiment de solidarité entre le peuple et les chefs nationalistes, comme il n'en exista jamais auparavant et même depuis. Seuls quelques politiciens, la presse et des membres du haut clergé n'étaient pas à l'unisson.

On ne peut mettre en doute la montée de la très forte opposition du Québec à la conscription, comme l'ont démontré les enquêtes Gallup, d'usage nouveau au Canada à cette époque. A la mi-mars, $21 \%$ de la population se prononçait pour la conscription alors que $79 \%$ était contre; le 11 avril, les résultats donnaient $26 \%$ pour et $74 \%$ contre; et à la fin d'avril, juste avant le vote, il n'y avait plus que $19 \%$ pour par rapport à une énorme majorité s'élevant à $81 \%$ contre la conscription pour le service militaire outre-mer. Le Gallup démontra aussi que, dans l'ensemble du Canada, les gens à revenus élevés favorisaient davantage la conscription que les membres de la classe ouvrière; ainsi en était-il des urbains comparativement aux ruraux. ${ }^{48}$

43 Fondation Lionel-Groulx, Fonds LPDC.

44 APC, Fonds L.-P. Picard, 24 avril 1942.

45 Fondation Lionel-Groulx, Fonds LPDC.

46 André Laurendeau, "Nous ne raserons pas la muraille", L'Action nationale, XIX (janvier 1942): 7-10.

47 Ibid., XIX (février-mars 1942) : 86-105. Il s'agit du titre de l'article de François-Albert Angers. - Pour une excellente analyse de l'opinion de la population de la ville de Québec, voir le rapport du consul américain, "The Political Outlook in Quebec", 11 mars 1942, copie aux U.S. National Archives, State Department, 842.00/634. 488-489.

48 Chiffres publiés dans le Public Opinion Quarterly (automne 1942): 
$\mathrm{Du}$ point de vue du gouvernement, la question de l'organisation constituait un aspect important du problème. Comment le plébiscite, ce moyen de préparer les gens à la conscription, serait-il organisé ? Y aurait-il une immense campagne de publicité soutenue par une pléiade d'orateurs ? Rien ne fut décidé avant le début d'avril, un délai qu'on s'explique difficilement même en tenant compte du fait que la loi qui autorisait le plébiscite ne fut acceptée en troisième lecture que le 4 mars. ${ }^{49} \mathrm{Il}$ avait été prévu que les ministres du Québec dirigeraient la campagne dans la province, tandis que le secrétaire d'Etat, Norman McLarty, assumerait la responsabilité de l'organisation dans le reste du Canada. Cependant il n'y avait aucune liaison entre McLarty et les ministres québécois et tout devint confus. D'ailleurs, au Québec, seuls les ministres menèrent une campagne active; pratiquement aucun des rares députés qui étaient en faveur du oui ne participa à la campagne.50 Comme le nota T. C. Davis, sous-ministre des Services nationaux de guerre, chargé de venir en aide à l'inepte McLarty: "We have been permitted only to spend public money to urge people to get out to vote. The stand was taken that to urge them to get out and vote affirmatively and spend public money therefore, would be improper, and that if it were done a fair appropriation should be made to those who would want to advertise a "No" vote, and that this would not be in the national interest." 51 Conséquemment, on dut entreprendre une souscription publique pour obtenir des fonds en vue d'un battage publicitaire pour le oui. Les dons provenant de cette souscription étaient déductibles pour fins d'impôt,, ${ }^{52}$ ce qui n'était pas prévu, semble-t-il, pour les contributions à la LPDC.

La tâche était trop lourde pour de simples députés. Alors que quelques-uns, comme Brooke Claxton, créaient des organisations non partisanes impressionnantes et consultaient des agences de publicité, ${ }^{53}$ d'autres, par contre, ne faisaient rien du tout ou très peu. Dans les grandes villes, on tenta de coordonner

49 Fonds King, t. 32, King à W. T. R. Preston, 31 mars 1942.

50 Fonds Moffat, Mémorandum d'une conversation avec T. C. Davis, 21 avril 1942. - King, Journal, 1er avril 1942, 275-276; 10 avril 1942, 301. - APC, Fonds Ian Mackenzie, dossier 526-21, McLarty à Mackenzie, 24 mars 1942.

51 Quartier général des Forces canadiennes, section historique, no 951.059 (D2), T. C. Davis à T. W. L. MacDermot, 24 avril 1942.

52 Queen's University, Fonds Norman Lambert, Journal, 27 avril 1942. - A titre d'exemples de dons sous forme de publicité, voir Saturday Night (25 avril 1942): 9, 13, 40.

53 Fonds Claxton, t. 152, Mémorandum, 10 avril 1942. 
les efforts; dans les régions rurales, la campagne pour le oui ne fit guère impression.

Durant la campagne menée au Québec, on mit l'accent sur l'idée que l'on pouvait s'en remettre en toute confiance à Mackenzie King pour ne pas dépasser la mesure. "N'oublions pas que le Gouvernement en général et que tout particulièrement notre premier ministre, dont le sain canadianisme est bien connu, n'ont aucun intérêt à demander au Canada des sacrifices qui ne seraient pas nécessaires." "AYEZ DONC CONFIANCE EN M. MACKENZIE KING ET VOTEZ OUI LE 27 AVRIL 1942" 54. Le Comité national du plébiscite à Ottawa envoya à tous les députés une masse de documents dont une longue liste des objections possibles accompagnées des réponses lénifiantes pour convaincre les électeurs de voter oui; ${ }^{55}$ mais une bonne partie de cette documentation ne fut jamais utilisée par les députés déçus. ${ }^{56}$

La campagne en faveur du oui, menée d'une façon si maladroite, eut pour effet de priver le gouvernement fédéral d'appuis influents au Québec. Malcolm Macdonald, haut-commissaire de la Grande-Bretagne à Ottawa, nota ce fait très clairement dans une dépêche qu'il envoya à Londres:

The Government had influential friends in Quebec who would have been ready to help, if Ministers had given them a clear lead. When I visited Quebec City at the end of February Cardinal Villeneuve... told me that at a judicious moment he and the Roman Catholic Archbishops would publish a "helpful statement". This naturally would have made a deep impression on the population... Again, the Premier of Quebec, Mr. Adelard Godbout, assured me that, though he could not make the first move, he and his colleagues in the Provincial Cabinet would give support to the Federal Government and try to persuade their fellow-citizens to vote "Yes" after the Government itself had opened its campaign. But ... the Government never did open its campaign until several weeks later. By then the vast majority of the people had been recruited into the other camp. It was too late for the Cardinal and the Roman Catholic hierarchy to ask their followers to fall in and march in the opposite direction. It was more than M. Godbout's political life was worth to issue the order "About turn". The best thing that the Cardinal and he could do was to refrain from authorising them to

54 Fonds Picard.

55 Ibid., 15 avril 1942.

56 Pour des exemples de désaffection, voir King, Journal, 4 février 1942,$113 ; 26$ mars 1942, 262-263. 
vote "No". Eventually Cardinal Villeneuve announced that the people should vote "according to their consciences", and the Premier produced some equally non-committal formula. 57

Malgré les difficultés croissantes concernant le vote au Québec, Mackenzie King se montra optimiste à la veille du plébiscite quand il rencontra Bruce Hutchison et Grant Dexter, les deux reporters-vedettes des journaux Sifton - deux hommes avec qui King avait des relations particulièrement étroites. A plusieurs reprises, d'après le long mémorandum de Dexter, le premier ministre fit des plaisanteries aux dépens de Ralston et aussi à propos des avis qu'il recevait de l'état-major. "I have talked to him again and again, dit King. I have asked him not once but many times why he does not tell the generals what we, the cabinet, think instead of continually telling us what the generals thinks [sic]. Generals, poursuivit-il, are almost invariably wrong." Même la tournure du plébiscite s'annonçait bien, d'ajouter le premier ministre. "The people who had opposed the plebiscite so bitterly [dans le parti conservateur] were now, he was pleased to notice (seraphic smile), supporting it and urging an affirmative vote." Puis, dans une réflexion étonnante, que Dexter rapporta sans doute avec exactitude vu son honnêteté éprouvée comme journaliste, King confia à ses deux hôtes qu'obtenir la conscription tout en conservant le pays uni serait le plus grand triomphe de la guerre.58 On ne sait exactement ce qu'il voulait signifier. S'attendait-il à ce qu'il pourrait appliquer la conscription après le plébiscite ? Ou est-ce qu'il laissait entendre qu'après le plébiscite on pourrait appliquer la conscription sans diviser le pays ? C'est vraisemblablement cette deuxième possibilité; mais néanmoins ses remarques ne laissent pas d'être étonnantes. De toute façon, les résultats du plébiscite devaient démontrer que King avait divagué.

Selon François-Albert Angers, lors du plébiscite on eut "un vote de race". 59 En général, les Canadiens français votèrent non et les Canadiens anglais votèrent yes, et ce sans aucune équivoque. L'Ontario vota yes à $82.3 \%$ et le Québec vota non à $72.9 \%$. On doit signaler cette chose remarquable que, dans les régions du pays où le vote allait à l'encontre de la tendance dominante,

57 Public Record Office, London, DO35/586, no 463, 4 août 1942.

58 Fonds Dexter, Mémorandum, 28 février 1942. - Le 25 mars 1942 Léopold Richer écrivit à Georges Pelletier que King avait dit au caucus libéral que la conscription était inévitable, Fondation Lionel-Groulx, Fonds Georges-Pelletier.

59 L'Action nationale, XIX (mai 1942) : 299-312. - King interpréta aussi les résultats comme "un vote de race", Pickersgill, ibid., I: 364. 
la composition ethnique était très particulière. Au Québec, dans neuf circonscriptions électorales, il y eut une nette majorité en faveur du yes. Toutes étaient situées dans la région de Montréal avec des majorités anglophones et cinq d'entre elles avaient des députés anglophones. Parmi les huit circonscriptions en dehors du Québec où l'on vota no, six étaient à forte prédominance canadienne-française et représentées par des députés francophones (Prescott et Russell en Ontario, Provencher au Manitoba, Gloucester, Restigouche-Madawaska et Kent au Nouveau-Brunswick). Les deux autres étaient principalement peuplées par des citoyens d'origine autre que française ou anglaise* (Vègreville en Alberta et Rosthern en Saskatchewan)..$^{60}$

Sans aucun doute les résultats au Québec étaient un triomphe pour la LPDC, un tribut à son énergie et à sa superbe organisation. Même Laurendeau fut étonné par l'importance de la victoire. "Le résultat, écrivit-il à Philippe Hamel, a dépassé nos espérances; je ne croyais pas qu'une majorité de NON pût être obtenue dans le comté de Chubby Power." 61 Le ministre de l'Aviation ne fut pas le seul à subir un échec dans sa tentative de rallier les électeurs en faveur du gouvernement. Dans les circonscriptions électorales de chaque ministre francophone du cabinet, il y eut une majorité contre le gouvernement, un refus total de la politique de King et de son appel à la compréhension.

Nonobstant les résultats du plébiscite, quelques Canadiens français ont dû garder leur confiance à Mackenzie King; mais la situation était très décourageante pour le premier ministre. Il avait tenu en échec ses adversaires au Canada anglais, il les avait même humiliés en les contraignant à travailler dans le sens de sa politique. Mais au Québec ce fut une tout autre histoire. Malgré tout, le plébiscite avait donné carte blanche au gouvernement et la volonté du peuple serait respectée ... peutêtre. "I felt very strongly that to keep Canada united, écrivit-il dans son journal, we would have to do all in our power [to keep] from reaching the point where necessity for conscription overseas would arise." Le plébiscite n'avait donc rien changé sauf que, si la conscription devenait nécessaire, on pourrait y recourir, "with the will of the majority, expressed in advance, and if proceedings are taken in the right way, will be gradually acquiesced in by those in the minority" 62 .

*Traduction du terme anglais "ethnic". vol. I.

60 The Canada Gazette, 23 juin 1942 et The Census of Canada 1941,

61 Fondation Lionel-Groulx, Fonds LPDC, 29 avril 1942.

62 Pickersgill, ibid., I: 364. 
Les jugements de King étaient très souvent justes, mais il n'avait pas prévu la lutte acharnée qui déchirerait son cabinet en mai, juin et juillet 1942, quand les ministres se disputèrent sur les implications du plébiscite. La Ligue pour la défense du Canada avait mis King et ses ministres dans une position difficile. Toutefois la conscription pour le service militaire outre-mer ne viendrait pas avant la fin de l'année 1944 et alors même elle serait très limitée. Pour l'essentiel King respecta ses promesses de 1939 et de 1940, quoique non sans difficulté et en donnant l'impression au Québec qu'il les avait reniées. The performance was as good as the promise. Malheureusement le plébiscite avait demandé au Canada tout entier de libérer le gouvernement d'une promesse faite au Québec seul; et le résultat de cette trahison ne servit qu'à affaiblir la confiance entre le gouvernement et le peuple, entre le Canada anglais et le Canada français.

(Traduction) 